\title{
EXTENSIONS OF CERTAIN DYNAMICAL THEOREMS OF HALPHEN AND KASNER
}

\author{
JOHN DECICCO
}

1. Introduction. We wish to present here some extensions of certain theorems of Halphen and Kasner concerning the dynamical trajectories of positional fields of force in space.

Kasner has developed the differential geometry of the dynamical trajectories of general positional fields of force in the plane and in space in his Princeton Colloquium Lectures. ${ }^{1}$ Recently Kasner and the author have introduced the concept of generalized fields of force which depend not only upon the position of the point but also upon the direction through the point. ${ }^{2}$ In a generalized field of force in space, there are $\infty^{5}$ dynamical trajectories just as in the ordinary positional case.

The theorem of Halphen which we wish to extend to generalized fields of force is that all positional fields of force whose $\infty^{5}$ dynamical trajectories are plane curves are of the central or parallel type. That is, the lines of force are all straight lines through a fixed point $O$ (which may be finite or at infinity). Moreover the $\infty^{5}$ trajectories consist of the $\infty^{2}$ systems of $\infty^{3}$ ordinary plane dynamical trajectories of the central or parallel type, each such system lying in a plane through the point $O$. There are $\infty^{3+f(3)}$ such systems of $\infty^{5}$ dynamical trajectories, ${ }^{3}$ and the number of central fields of force is $\infty^{4+f(3)}$.

We find that all generalized fields of force whose $\infty^{5}$ dynamical trajectories are plane curves form a more extensive class. The $\infty^{5}$ trajectories consist, in general, of $\infty^{2}$ systems of $\infty^{3}$ generalized plane dynamical trajectories, each such system lying in a plane tangent to a base surface $\Sigma$. The various degenerate situations are four in number and may be described as follows: (i) The base planes are tangent to a given curve $C$, (ii) the base planes pass through a given finite point $O$, (iii) the base planes are all parallel to the tangent planes of a given

Presented to the Society, April 23, 1943; received by the editors January 2, 1943, and, in revised form, May 4, 1943.

${ }^{1}$ Kasner, Differential geometric aspects of dynamics, Amer. Math. Soc. Colloquium Publications, vol. 3, 1913, 1934. Also see Trans. Amer. Math. Soc. vols. 7-11 (19061910).

${ }^{2}$ See (1) A generalized theory of dynamical trajectories, Trans. Amer. Math. Soc. vol. 54 (1943) pp. 23-38; and (2) Generalized dynamical trajectories in space abstract 49-3-120.

${ }^{3}$ The symbol $\infty^{k f(n)}$ denotes the content of a geometric manifold which depends on $k$ functions of $n$ variables only. See Kasner, $A$ notation for infinite manifolds, Amer. Math. Monthly vol. 49 (1942) pp. 243-244. 
cone $\Gamma$, and (iv) the base planes are all parallel to a fixed line $L$. The number of such planar systems of generalized dynamical trajectories is $\infty f(2)+2 f(5)$, and the number of associated generalized fields of force is $\infty f(2)+f(4)+2 f(5)$

The theorem of Kasner which we wish to generalize is concerned with arbitrary positional fields of force and may be stated in the following manner. If a particle starts at rest from any given point $O$ the resulting rest trajectory which it describes will be initially tangent to, and also will possess the same osculating plane as, the line of force at $O$; moreover, the ratio of the curvature of the rest trajectory to that of the line of force is $1: 3$.

In a generalized field of force this theorem is no longer valid. It is true that the rest trajectory and the line of force will be initially tangent, but, in general, they will have different osculating planes at $O$. We determine all those fields of force such that, at any point $O$ of space, the rest trajectory and the line of force will have the same osculating plane. In the final part of our paper, we obtain a converse of Kasner's theorem.

2. The differential equations of the $\infty^{5}$ generalized dynamical trajectories. We now consider any generalized field of force in space. Observe that an ordinary positional field of force could be called isotropic whereas a generalized field of force could be termed anisotropic. (A state of stress in a solid medium is an example of a generalized field of force, although it is not of the most general character.)

Let $F(\phi, \psi, \chi)$ be the rectangular components of the generalized force vector which corresponds to the lineal element $E\left(x, y, z, y^{\prime}, z^{\prime}\right)$. (Note that the primes denote differentiation with respect to $x$ ). We assume that the direction of our force vector $F(\phi, \psi, \chi)$ does not identically agree with that of the corresponding lineal element $E$. The equations of motion of a particle of unit mass are

$$
\begin{gathered}
d^{2} x / d t^{2}=\phi\left(x, y, z, y^{\prime}, z^{\prime}\right), \quad d^{2} y / d t^{2}=\psi\left(x, y, z, y^{\prime}, z^{\prime}\right), \\
d^{2} z / d t^{2}=\chi\left(x, y, z, y^{\prime}, z^{\prime}\right),
\end{gathered}
$$

where, of course, $t$ is the time.

Upon eliminating the time $t$ from these equations, it is found that the $\infty^{5}$ dynamical trajectories of a generalized field of force are given as the integral curves of either of the two Monge differential equations of third order

$$
\begin{aligned}
\left(\psi-y^{\prime} \phi\right) y^{\prime \prime \prime}= & {\left[\left(\psi_{x}+y^{\prime} \psi_{y}+z^{\prime} \psi_{z}\right)-y^{\prime}\left(\phi_{x}+y^{\prime} \phi_{y}+z^{\prime} \phi_{z}\right)\right] y^{\prime \prime} } \\
& +\left[\psi_{y^{\prime}}-y^{\prime} \phi_{y^{\prime}}-3 \phi\right] y^{\prime \prime 2}+\left(\psi_{z^{\prime}}-y^{\prime} \phi_{z^{\prime}}\right) y^{\prime \prime} z^{\prime \prime}
\end{aligned}
$$


or

$$
\begin{aligned}
\left(\chi-z^{\prime} \phi\right) z^{\prime \prime \prime}= & {\left[\left(\chi_{x}+y^{\prime} \chi_{y}+z^{\prime} \chi_{z}\right)-z^{\prime}\left(\phi_{x}+y^{\prime} \phi_{y}+z^{\prime} \phi_{z}\right)\right] z^{\prime \prime} } \\
& +\left[\chi_{z^{\prime}}-z^{\prime} \phi_{z^{\prime}}-3 \phi\right] z^{\prime \prime 2}+\left(\chi_{y^{\prime}}-z^{\prime} \phi_{y^{\prime}}\right) y^{\prime \prime} z^{\prime \prime}
\end{aligned}
$$

solved together with the Monge differential equation of second order

$$
z^{\prime \prime}=\left[\left(\chi-z^{\prime} \phi\right) /\left(\psi-y^{\prime} \phi\right)\right] y^{\prime \prime} .
$$

Therefore the generalized dynamical trajectories are of the threedimensional type $(\mathrm{G})$, namely

$$
\begin{aligned}
z^{\prime \prime} & =K\left(x, y, z, y^{\prime}, z^{\prime}\right) y^{\prime \prime}, \\
y^{\prime \prime \prime} & =G\left(x, y, z, y^{\prime}, z^{\prime}\right) y^{\prime \prime}+H\left(x, y, z, y^{\prime}, z^{\prime}\right) y^{\prime \prime 2} .
\end{aligned}
$$

Conversely any system of $\infty^{5}$ curves of the three-dimensional type (G) represents the dynamical trajectories of $\infty^{f(4)}$ generalized fields of force.

Kasner has shown that the three-dimensional type (G) may be characterized by the two geometric properties listed below. For each of the $\infty^{1}$ trajectories passing through a given lineal element $E$, construct the osculating plane and the osculating sphere at $E$. The two properties are:

Property I. The $\infty^{1}$ trajectories possess the same osculating plane at $E$.

Property II. The locus of the centers of the osculating spheres at $E$ is a straight line.

3. Extension of Halphen's theorem. Now we shall discuss our extension of the Halphen problem.

Theorem 1. A system of $\infty^{5}$ generalized dynamical trajectories consists wholly of plane curves if and only if it is represented by a system of differential equations of the three-dimensional type $(\mathrm{G})$ where the function $K$ is defined as a solution of an equation of the form

$$
F\left[z^{\prime}-y^{\prime} K, K, z-x\left(z^{\prime}-y^{\prime} K\right)-y K\right]=0,
$$

where $F$ is an arbitrary function of three variables.

There are thus $\infty f(2)+2 f(5)$ systems of generalized dynamical trajectories which consist wholly of plane curves.

We proceed with the proof of Theorem 1. Upon substituting the three-dimensional type (G) into the condition $y^{\prime \prime} z^{\prime \prime \prime}-y^{\prime \prime \prime} z^{\prime \prime}=0$ for plane curves and simplifying, we obtain the equation

$$
y^{\prime \prime 3}\left(K_{y^{\prime}}+K K_{z^{\prime}}\right)+y^{\prime \prime 2}\left(K_{x}+y^{\prime} K_{y}+z^{\prime} K_{z}\right)=0 .
$$


By this equation we see that, upon excluding the $\infty^{4}$ straight lines which are trajectories of any three-dimensional type $(\mathrm{G})$, there are at most $\infty^{4}$ trajectories in any system of $\infty^{5}$ dynamical trajectories, which are plane curves.

Now if our three-dimensional type $(G)$ is to contain more than $\infty^{4}$ plane trajectories, we observe that (5) must be an identity in $y^{\prime \prime}$. Therefore the function $K$ must satisfy the two partial differential equations of the first order

$$
K_{y^{\prime}}+K K_{z^{\prime}}=0, \quad K_{x}+y^{\prime} K_{y}+z^{\prime} K_{z}=0 .
$$

First let us suppose that $K$ is independent of $y^{\prime}$ or $z^{\prime}$. From these equations we see that $K$ must be constant. This solution for $K$ is given by the equation (4) where $F$ is a function of the second variable $K$ alone. Therefore, we can henceforth suppose that $K$ contains $y^{\prime}$ or $\boldsymbol{z}^{\prime}$ explicitly.

By the first of equations (6) and by the fact that $K$ contains $y^{\prime}$ or $z^{\prime}$, we find that $K$ must be a solution of a finite equation of the form

$$
z^{\prime}-y^{\prime} K=\lambda(K, x, y, z),
$$

where $\lambda$ is an arbitrary function of four variables. ${ }^{4}$ Obtaining the partial derivatives of $K$ with respect to $x, y$, and $z$ from this equation and substituting into the second equation of (6), we find

$$
\lambda_{x}+y^{\prime} \lambda_{y}+z^{\prime} \lambda_{z}=0 \text {. }
$$

Eliminating $z^{\prime}$ from this equation and equation (7), we find

$$
y^{\prime}\left(\lambda_{y}+K \lambda_{z}\right)+\left(\lambda_{x}+\lambda \lambda_{z}\right)=0 .
$$

Now this must be an identity in $y^{\prime}$. Assume the contrary. We then can solve this equation for $y^{\prime}$ and differentiate the result with respect to $z^{\prime}$. Since, by (7), $K_{z^{\prime}} \neq 0$ we find

$$
\frac{\partial}{\partial K}\left(\frac{\lambda_{x}+\lambda \lambda_{z}}{\lambda_{y}+K \lambda_{z}}\right)=0 .
$$

This will mean that the equation (9) solved for $y^{\prime}$ is independent of $y^{\prime}$. This is absurd. Hence (9) is an identity in $y^{\prime}$.

By the preceding argument, the function $\lambda$ of $(K, x, y, z)$ must satisfy the two partial differential equations of the first order

$$
\lambda_{y}+K \lambda_{z}=0, \quad \lambda_{x}+\lambda \lambda_{z}=0 .
$$

${ }^{4}$ This equation (7) arises in the characterization of ordinary positional fields of force in space. See Kasner, Dynamical trajectories: The motion of a particle in an arbitrary field of force, Trans. Amer. Math. Soc. vol. 8 (1907) pp. 135-158. 
Suppose now that $\lambda_{z}=0$. These equations show that $\lambda$ is independent of $(x, y, z)$. Therefore $\lambda$ is a function of $K$ only. Substituting this result into (7), we discover that the function $K$ is given by the equation (4) where $F$ is independent of the third variable. Henceforth we may suppose that $\lambda_{z} \neq 0$.

Since $\lambda_{z} \neq 0$, we find from the second of equations (11) that $\lambda$ must satisfy an equation of the form

$$
z-x \lambda=\mu(\lambda, K, y),
$$

where $\mu$ is an arbitrary function of three variables. Obtaining the partial derivatives of $\lambda$ with respect to $y$ and $z$ from this equation, and substituting into the first of equations (11), we find

$$
\mu_{y}=K \text {. }
$$

The integration of this equation yields the following solution for $\mu$,

$$
\mu=y K+f(\lambda, K)
$$

where $f$ is an arbitrary function of two variables. Substituting this into (12), we find

$$
z=x \lambda+y K+f(\lambda, K) .
$$

Finally, eliminating $\lambda$ from this equation and equation (7), we discover that

$$
z=x\left(z^{\prime}-y^{\prime} K\right)+y K+f\left(z^{\prime}-y^{\prime} K, K\right) .
$$

This may be obtained as a solution of the equation (4) for the third variable. Thus the proof of Theorem 1 is complete.

To obtain Halphen's theorem as a consequence of Theorem 1, we first observe that, for the ordinary positional case, equation (3) demonstrates that equation (4) must be a function of a linear integral function in $\left(z^{\prime}-K y^{\prime}\right)$ and $K$ with coefficients functions of $(x, y, z)$ only. If the third variable is missing in (4), then the coefficients must be constants and the field of force is of the parallel type. On the other hand, if the third variable is present in (4), the equation may be written in the form (16), where $f$ is integral linear in the two variables with constant coefficients. Solving the resulting equation for $K$, we find that the field of force is of the central type. This completes the proof of Halphen's theorem.

Theorem 2. The $\infty^{5}$ generalized dynamical trajectories of Theorem 1 consist of $\infty^{2}$ systems of $\infty^{3}$ generalized plane dynamical trajectories, each such system lying in a plane which is, in general, tangent to a base surface $\Sigma$. 
The degenerate situations which arise are four in number and may be described as follows: (i) The base planes are tangent to a given curve $C$, (ii) the base planes pass through a given finite point $O$, (iii) the base planes are all parallel to the tangent planes of a given cone $\Gamma$, and (iv) the base planes are all parallel to a fixed line $L$.

To prove Theorem 2, we first observe that the differential equations of the dynamical trajectories of Theorem 1 may be written as

$$
\begin{gathered}
F\left[z^{\prime}-y^{\prime} z^{\prime \prime} / y^{\prime \prime}, z^{\prime \prime} / y^{\prime \prime}, z-x\left(z^{\prime}-y^{\prime} z^{\prime \prime} / y^{\prime \prime}\right)-y z^{\prime \prime} / y^{\prime \prime}\right]=0, \\
y^{\prime \prime \prime}=G y^{\prime \prime}+H y^{\prime \prime 2} .
\end{gathered}
$$

To find out what curves of this system (17) lie in the plane $z=a x+b y+c$, we eliminate $z$ between the first of equations (17) and the equation of the plane. The result of this elimination yields

$$
F(a, b, c)=0 \text {. }
$$

Therefore we conclude that for a plane to contain at least one curve of (17), its parameters $(a, b, c)$ must satisfy the preceding equation. Moreover any such plane will contain $\infty^{3}$ spatial generalized dynamical trajectories, and these form a generalized plane dynamical system of arbitrary character. The conclusion of our theorem is obtained from the observation that the $\infty^{2}$ planes whose parameters $(a, b, c)$ satisfy (18) are, in general, tangent to a surface $\Sigma$. The degenerate situations also can be obtained by a discussion of the various forms that the function $F$ may assume.

It must be noted here that any plane system of $\infty^{3}$ generalized dynamical trajectories of Theorem 2 is of perfectly general character, whereas in Halphen's theorem any such plane system must be of the central or parallel type.

4. The lines of force and the rest trajectories. In the remainder of this paper, we shall suppose that

$$
\left(\psi_{y^{\prime}}-y^{\prime} \phi_{y^{\prime}}-\phi\right)\left(\chi_{z^{\prime}}-z^{\prime} \phi_{z^{\prime}}-\phi\right)-\left(\psi_{z^{\prime}}-y^{\prime} \phi_{z^{\prime}}\right)\left(\chi_{y^{\prime}}-z^{\prime} \phi_{y^{\prime}}\right) \neq 0
$$

and

$$
\left(\psi_{y^{\prime}}-y^{\prime} \phi_{y^{\prime}}-3 \phi\right)\left(\chi_{z^{\prime}}-z^{\prime} \phi_{z^{\prime}}-3 \phi\right)-\left(\psi_{z^{\prime}}-y^{\prime} \phi_{z^{\prime}}\right)\left(\chi_{y^{\prime}}-z^{\prime} \phi_{y^{\prime}}\right) \neq 0 \text {. }
$$

Let us first of all consider the lines of force in a generalized field of force. At any point $O$ there is, in general, one (and at most $\infty^{1}$ ) lineal element $E_{0}$ whose direction coincides with that of the corresponding force vector $F_{0}$. The lines of force are the integral curves of the differential equations defined by all the lineal elements $E_{0}$ of a certain 
region of space. This system of differential equations is

$$
\psi-y^{\prime} \phi=0, \quad \chi-z^{\prime} \phi=0 .
$$

By (19.1), it follows that there are, in general, $\infty^{2}$ lines of force. Otherwise if the left-hand side of (19.1) is identically zero, there are $\infty^{f(1)}$, or $\infty^{1}$, or a finite number of lines of force.

Consider next the rest trajectories in a generalized field of force. A rest trajectory is defined as the trajectory described by a particle of unit mass which starts at rest from any point $O$. By (19.2) there are, in general, $\infty^{3}$ rest trajectories in a generalized field of force. Otherwise if the left-hand member of (19.2) is identically zero, there are at most $\infty^{f(1)}$ rest trajectories.

Thus it is obvious that under the assumptions (19.1) and (19.2) there are, in general, $\infty^{2}$ lines of force and $\infty^{3}$ rest trajectories.

5. The extension of Kasner's theorem concerning one-third of the curvatures. In this section we shall determine all generalized fields of force such that, at any point $O$, the rest trajectory and the line of force will have the same osculating plane.

In the first place, we can easily show that the rest trajectory through the point $O$ is tangent to the line of force at $O$. This is an immediate consequence of the equations

$$
\begin{aligned}
d y / d t=y^{\prime} d x / d t, & d^{2} y / d t^{2}=y^{\prime} d^{2} x / d t^{2}+y^{\prime \prime}(d x / d t)^{2}, \\
d z / d t=z^{\prime} d x / d t, & d^{2} z / d t^{2}=z^{\prime} d^{2} x / d t^{2}+z^{\prime \prime}(d x / d t)^{2},
\end{aligned}
$$

together with the initial conditions $(d x / d t)_{0}=(d y / d t)_{0}=(d z / d t)_{0}=0$ and the equations (20) defining the lines of force.

We discuss our extension of Kasner's theorem.

THEOREM 3. The rectangular components $F(\phi, \psi, \chi)$ of any generalized field of force for which, at any point $O$ of space, the rest trajectory and the line of force possess the same osculating plane at $O$ must be such that the single eliminant with respect to $y^{\prime}$ and $z^{\prime}$ of the three equations

$$
\psi-y^{\prime} \phi=0, \quad \chi-z^{\prime} \phi=0, \quad(A C+B F) /(D C+E F)=C / F,
$$

where $A, B, C, D, E, F$ are defined by the expressions

$$
\begin{array}{rr}
A & =\psi_{y^{\prime}}-y^{\prime} \phi_{y^{\prime}}, \quad B=\psi_{z^{\prime}}-y^{\prime} \phi_{z^{\prime}}, \\
C & =\left(\psi_{x}+y^{\prime} \psi_{y}+z^{\prime} \psi_{z}\right)-y^{\prime}\left(\phi_{x}+y^{\prime} \phi_{y}+z^{\prime} \phi_{z}\right), \\
D & =\chi_{y^{\prime}}-z^{\prime} \phi_{y^{\prime}}, \\
F=\chi_{z^{\prime}}-z^{\prime} \phi_{z^{\prime}}, & =\left(\chi_{x}+y^{\prime} \chi_{y}+z^{\prime} \chi_{z}\right)-z^{\prime}\left(\phi_{x}+y^{\prime} \phi_{y}+z^{\prime} \phi_{z}\right),
\end{array}
$$

is identically zero. 
In the first place, the first derivatives $\left(y^{\prime}, z^{\prime}\right)$ of the line of force and the rest trajectory through the point $O$ are given as solutions of the equations (20).

By obtaining the total derivatives of equations (20) with respect to $x$ and using the formulas (23), we find that the equations defining the second derivatives $\left(Y^{\prime \prime}, Z^{\prime \prime}\right)$ of the line of force through the point $O$ are

$$
(A-\phi) Y^{\prime \prime}+B Z^{\prime \prime}+C=0, \quad D Y^{\prime \prime}+(E-\phi) Z^{\prime \prime}+F=0 .
$$

By equations (2.1) and (2.2), we see that the equations defining the second derivatives $\left(y^{\prime \prime}, z^{\prime \prime}\right)$ of the rest trajectory through the point $O$ are

$$
(A-3 \phi) y^{\prime \prime}+B z^{\prime \prime}+C=0, \quad D y^{\prime \prime}+(E-3 \phi) z^{\prime \prime}+F=0 .
$$

It is obvious that, in general, the osculating planes can not be identical. Let us impose the condition that the two osculating planes be identical. Then there must exist a number $\rho$ such that

$$
y^{\prime \prime}=\rho Y^{\prime \prime}, \quad z^{\prime \prime}=\rho Z^{\prime \prime} .
$$

Eliminating $Y^{\prime \prime}$ and $Z^{\prime \prime}$ from equations (24), (25), and (26), we find

$$
\begin{aligned}
y^{\prime \prime} & =(1-\rho) C / 2 \phi, \quad z^{\prime \prime}=(1-\rho) F / 2 \phi, \\
\rho & =(A C+B F-\phi C) /(A C+B F-3 \phi C) \\
& =(D C+E F-\phi F) /(D C+E F-3 \phi F) .
\end{aligned}
$$

This last equation establishes the validity of Theorem 3 .

For the generalized fields of force of Theorem 3, let $\lambda$ be defined by the two equal expressions

$$
\lambda=(A C+B F) / \phi C=(D C+E F) / \phi F .
$$

THEOREM 4. For the generalized fields of force of Theorem 3 , the ratio $\rho$ of the curvature of the rest trajectory to that of the line of force is given by the formula

$$
\rho=(1-\lambda) /(3-\lambda) .
$$

This result may be considered to be an extension of Kasner's onethird theorem.

6. A converse of Kasner's theorem concerning one-third the curvatures. If, in addition to the possession of the same osculating plane, we impose the condition that the ratio $\rho=1 / 3$, we obtain the following converse of Kasner's theorem. 
THEOREM 5. The rectangular components $F(\phi, \psi, \chi)$ of any generalized field of force for which, at any point $O$ of space, the rest trajectory and the line of force possess the same osculating plane and the ratio $\rho$ of the curvature of the rest trajectory to that of the line of force is $1 / 3$ must be such that the two eliminants with respect to $y^{\prime}$ and $z^{\prime}$ of the four equations

$$
\begin{aligned}
\psi-y^{\prime} \phi & =0, & \chi-z^{\prime} \phi & =0, \\
A C+B F & =0, & D C+E F & =0,
\end{aligned}
$$

where $(A, B, C, D, E, F)$ are defined by equations (23), are identically zero.

Thus it is seen that, in the generalized fields of force in space, there are no general geometric relations between the lines of force and the rest trajectories except for the trivial one concerning the possession of the common tangent line. This is entirely different from the twodimensional case (see footnote 2) where there is a theory-the general theorem being quite similar to our Theorem 4 .

Illinois Institute of Technology 